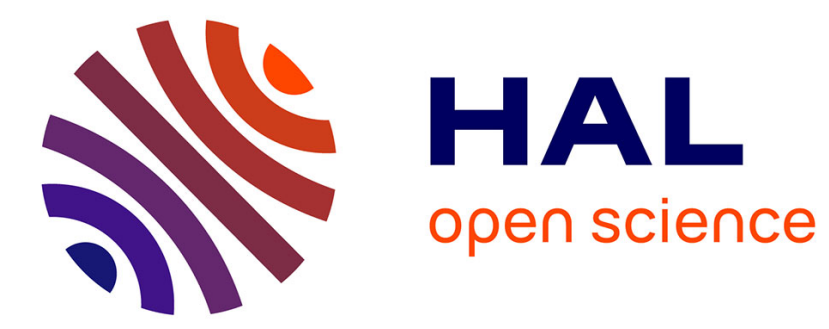

\title{
How Mobility Impacts the Performance of Inter-cell Coordination in Cellular Data Networks
}

\author{
Nivine Abbas, Thomas Bonald, Berna Sayrac
}

\section{To cite this version:}

Nivine Abbas, Thomas Bonald, Berna Sayrac. How Mobility Impacts the Performance of Inter-cell

Coordination in Cellular Data Networks. Globecom, 2015, San Diego, United States. hal-01245211

\author{
HAL Id: hal-01245211 \\ https://hal.inria.fr/hal-01245211
}

Submitted on 16 Dec 2015

HAL is a multi-disciplinary open access archive for the deposit and dissemination of scientific research documents, whether they are published or not. The documents may come from teaching and research institutions in France or abroad, or from public or private research centers.
L'archive ouverte pluridisciplinaire HAL, est destinée au dépôt et à la diffusion de documents scientifiques de niveau recherche, publiés ou non, émanant des établissements d'enseignement et de recherche français ou étrangers, des laboratoires publics ou privés. 


\section{How Mobility Impacts the Performance of Inter-cell Coordination in Cellular Data Networks}

\author{
Nivine Abbas \\ Orange Labs \& Telecom ParisTech \\ Paris, France \\ nivine.abbas@telecom-paristech.fr
}

\author{
Thomas Bonald \\ Telecom ParisTech \\ Paris, France \\ thomas.bonald@telecom-paristech.fr
}

\author{
Berna Sayrac \\ Orange Labs \\ Issy-Les-Moulineaux, France \\ berna.sayrac@orange.com
}

\begin{abstract}
In this paper, we assess the performance of intercell coordination in the presence of mobility. This performance depends primarily on the resource allocation scheme. Indeed, a scheduling strategy which may seem efficient when users are static can lead to bad performance when users are mobile. Several scheduling policies are investigated. Their performance critically depends on their ability to predict users' mobility. The results are based on the analysis of flow-level traffic models.
\end{abstract}

Keywords-Cellular data networks, mobility, sector coordination, flow-level modeling, queuing theory.

\section{INTRODUCTION}

Inter-cell interference is one of the key challenges faced in mobile communication systems. It restricts the re-usability of the radio resource and limits spectral efficiency. Since the days of GSM, various techniques are used to cope with interference. Coordinated MultiPoint (CoMP) [1], [2], a main feature on the LTE-A roadmap, is mentioned as a promising approach to mitigate its effects through the coordination of multiple cells.

In this paper, we focus on joint processing (JP) schemes, which can be achieved through two main techniques: joint transmission (JT) and dynamic point selection (DPS). JT consists in transmitting data simultaneously from multiple cells to a single user; DPS consists in dynamically selecting the best transmitting cell in the coordination cluster and in muting the other cells. Under both JT and DPS, all cells of the coordination cluster are involved in the transmission and thus not available for other users. Thus there is a tradeoff between the performance of cell-edge users and the ability of the network to process all traffic.

It is well known that CoMP schemes are only advantageous for cell-edge users [3]. It has recently been shown that they can also be detrimental for cell-edge users at high load, due to the inefficient utilization of radio resources [4]. Their efficiency in fact depends critically on the scheduling strategy. It is proposed in [5], [6] to allocate a dedicated frequency band to cell-edge users in order to perform CoMP operations. In [7], the authors propose a joint proportional fairness scheduling algorithm that treats cell-center and cell-edge users equally without any frequency band partitioning. The scheduling algorithm may also depend on the clustering technique, from static clusters with centralized scheduling [8] to dynamic clusters with distributed scheduling [9].

It is common to assume static users in the performance evaluation of CoMP schemes. We show in this paper that mobility has a strong impact on the performance of CoMP schemes. A scheduling strategy which may seem efficient when users are static can lead to bad performance when users are mobile. This is a rather surprising result since mobility is generally thought as improving throughput performance, see for instance [10], [11], [12], [13], [14], [15], [16]. We study the impact of mobility on the performance of CoMP under several scheduling strategies. The results are based on the analysis of flow-level traffic models.

The rest of the paper is organized as follows. In the next section, we present the reference model under various scheduling schemes in the absence of mobility. We then analyze the impact of mobility and conclude the paper.

\section{REFERENCE MODEL}

We first present the reference model in the absence of mobility under various scheduling schemes: a static allocation, the proportional fair allocation and two prioritization strategies.

\section{A. Cellular network}

We consider a cluster composed of $K$ coordinated cells, where each cell is modeled by two main zones: a noncoordination zone and a coordination zone, which is part of the global coordination zone of the cluster. The non-coordination zones $k=1, \ldots, K$, as illustrated by Figure 1 , are those where users are typically close to the cell center, experience good radio conditions and thus served only by their own serving base station. The coordination zone $(k=0)$ is that where users are close to the cell edge, suffer from bad radio conditions and receive signals from the $K$ coordinated cells. This corresponds to the JT scheme. In fact, the model applies to the DPS scheme as well since the $K$ coordinated cells are involved in the transmission in both cases; only the transmission rates achieved by the coordination depend on the JP scheme, either JT or DPS.

We model each zone $k$ by a set of $N_{k}$ regions. In each region, radio conditions are supposed to be homogeneous and thus users are served at the same physical data rate on the downlink. We model each region by a queue with a specific service rate corresponding to the physical data rate in this region. Consequently, the considered cluster can be viewed as a set of $\sum_{k=0}^{K} N_{k}$ queues with $K$ coupled processors. The simple case of two coordinated cells and one region in each zone is illustrated by Figure 1. Figure 2 shows an example of radio resource sharing between CoMP and non-CoMP users. 


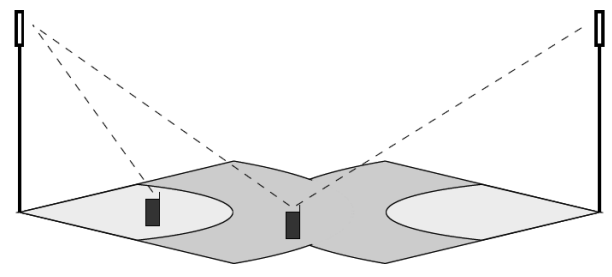

Fig. 1. A simple model with two coordinated cells.

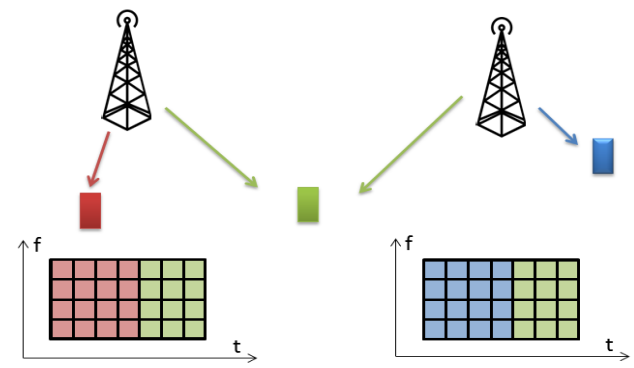

Fig. 2. Resource allocation between CoMP and non-CoMP users.

\section{B. Traffic model}

We consider elastic traffic only, corresponding to data transfers. Specifically, we assume that new data flows are generated in region $i$ of zone $k$ at the random times of a Poisson process of intensity $\lambda_{i k}$. We denote by $\lambda_{k}=\sum_{i} \lambda_{i k}$ the total flow arrival rate in zone $k$ and by $\lambda=\sum_{k} \lambda_{k}$ the total flow arrival rate in the coordination cluster. The probability that a new data flow is generated by a user in zone $k$ is $p_{k}=\lambda_{k} / \lambda$ while the probability that a new flow in zone $k$ is generated by a user in region $i$ is $p_{i k}=\lambda_{i k} / \lambda_{k}$. Physically, $p_{k}$ corresponds to the relative area of zone $k$ compared to the area of the cluster, while $p_{i k}$ represents the relative area of region $i$ of zone $k$ compared to the area of zone $k$, assuming uniform traffic distribution in the cell.

We use a flow-level model where each data flow is viewed as a fluid of random volume to be transmitted. The volumes have an exponential distribution with mean $\sigma$ (in bits). When region $i$ of zone $k$ is served, flows are completed at rate $\mu_{i k}$ in the absence of fast fading, corresponding to the physical rate $\mu_{i k} \sigma$ (in bit/s).

We denote by $X_{i k}(t)$ the number of active flows in region $i$ of zone $k$ at time $t$ and by $X_{k}(t)=\sum_{i} X_{i k}(t)$ the total number of active flows in zone $k$. The vector $X(t)=\left(X_{i k}(t)\right)_{i k}$ defines a Markov process of dimension $\sum_{k} N_{k}$. The load of region $i$ in zone $k$ is $\rho_{i k}=\lambda_{i k} / \mu_{i k}$. The total load of zone $k$ is given by $\rho_{k}=\sum_{i} \rho_{i k}=\lambda_{k} / \mu_{k}$, where $\mu_{k}$ is the weighted harmonic mean service rate:

$$
\mu_{k}=\frac{1}{\sum_{i} p_{i k} / \mu_{i k}} .
$$

The resources of each cell are shared between CoMP users (in coordination zone $k=0$ ) and non-CoMP users (in noncoordination zones $k=1, \ldots, K)$, as illustrated by Figure 2 . The actual service rate in zone $k$ is modulated by $\phi_{k}$, the fraction of time spent by the scheduler on users in zone $k$. This depends on the system state $x$ and on the scheduling policy.
For work-conserving policies, we have

$$
\forall k: x_{k}>0, \quad \phi_{k}(x)+\phi_{0}(x)=1 .
$$

We focus on how to allocate resources between CoMP and non-CoMP users' data flows. In each zone, users are assumed to share the allocated radio resources equally, independently of their radio conditions. However, when the network operates under an opportunistic scheduler which exploits the fast fading, a rate gain [10] should be taken into account in each region of each zone. Note that this gain depends on the number of flows in the considered region.

A necessary condition for the ergodicity of the Markov process $X(t)$ is $\rho<1$ in the absence of mobility, with

$$
\rho=\rho_{0}+\max _{k=1, \ldots, K} \rho_{k},
$$

that is $\rho<\lambda / \mu$ with

$$
\mu=\left(\frac{p_{0}}{\mu_{0}}+\max _{k=1, \ldots, K} \frac{p_{k}}{\mu_{k}}\right)^{-1} .
$$

The sufficient stability condition depends on the scheduling policy. We shall see in the following sections that mobility may increase or decrease the stability region, depending on the scheduling strategy.

\section{Throughput metrics}

We measure performance in terms of mean throughputs in the different zones. In any state $x$ such that $x_{i k}>0$, each user in region $i$ of zone $k$ has throughput $\mu_{i k} \sigma \phi_{k}(x) / x_{k}$. Now the distribution seen by users in region $i$ of zone $k$ is the sizebiased distribution [17]:

$$
\pi_{i k}(x) \propto x_{i k} \pi(x) .
$$

We denote by $E_{i k}$ the corresponding expectation. The mean throughput of users in region $i$ of zone $k$ is then given by

$$
\gamma_{i k}=E_{i k}\left(\frac{\mu_{i k} \sigma \phi_{k}(X)}{X_{k}}\right)=\frac{E\left(\mu_{i k} \sigma \phi_{k}(X) \frac{X_{i k}}{X_{k}}\right)}{E\left(X_{i k}\right)} .
$$

By the traffic conservation equation

$$
\lambda_{i k}=E\left(\mu_{i k} \phi_{k}(X) \frac{X_{i k}}{X_{k}}\right)
$$

we deduce

$$
\gamma_{i k}=\frac{\lambda_{i k} \sigma}{E\left(X_{i k}\right)}
$$

This is the ratio of the traffic intensity in region $i$ of zone $k$ to the mean number of data flows in this region. Observe that, by Little's law, this is also the ratio of mean flow size $\sigma$ to mean flow duration in region $i$ of zone $k$.

By a similar argument, we obtain the mean throughput in zone $k$,

$$
\gamma_{k}=\frac{\lambda_{k} \sigma}{E\left(X_{k}\right)}
$$

and the mean throughput in the cluster,

$$
\gamma=\frac{\lambda \sigma}{E\left(\sum_{k} X_{k}\right)}
$$

This is the harmonic mean of the mean throughputs $\gamma_{k}$ weighted by $p_{k}$, for $k=0,1, \ldots, K$. 


\section{Static allocation}

A simple strategy consists in allocating a fixed fraction of resources to CoMP and non-CoMP users' data flows, as proposed by 3GPP in [5], [6]: a CoMP frequency sub-band is introduced to perform CoMP operation, so that all users selected to be served in CoMP mode use this pre-configured frequency sub-band. CoMP users' data flows are allocated some fixed fraction $\phi_{0}$ of the resources of each coordinated cell while non-CoMP users' data flows in cell $k$ are allocated the fraction $\phi_{k}=1-\phi_{0}$ of the considered cell radio resources.

The corresponding Markov process $X(t)$ is reversible, with stationary distribution

$$
\pi(x)=\prod_{k=0}^{K} \frac{\left(1-\rho_{k} / \phi_{k}\right)}{\phi_{k}^{x_{k}}}\left(\begin{array}{c}
x_{k} \\
x_{1 k}, \ldots, x_{N_{k} k}
\end{array}\right) \rho_{1 k}^{x_{1 k}} \ldots \rho_{N_{k} k}^{x_{N_{k} k}},
$$

provided $\rho_{0}<\phi_{0}$ and $\rho_{k}<1-\phi_{0}$ for all $k=1, \ldots, K$. Observe that this condition may be more restrictive than the natural stability condition $\rho<1$.

From (5), the mean throughput in zone $k$ is

$$
\gamma_{k}=\phi_{k} \mu_{k} \sigma\left(1-\rho_{k} / \phi_{k}\right)
$$

Observe that the mean throughput in zone $k$ decreases linearly from the physical rate $\phi_{k} \mu_{k} \sigma$ to 0 when the load of the corresponding zone increases from 0 to $\phi_{k}$. Indeed, the stability condition of zone $k$ is $\rho_{k}<\phi_{k}$.

\section{E. Proportional fairness}

Under proportional fairness [18], resources are shared so as to maximize

$$
\sum_{k=0}^{K} x_{k} \log \phi_{k}
$$

We get

$$
\phi_{0}(x)=\frac{x_{0}}{\sum_{k=0}^{K} x_{k}}
$$

and $\phi_{k}(x)=1-\phi_{0}(x)$ for all $k$ and all $x$ such that $x_{k}>0$.

We generalize the model presented in [4] for $K=2$ coordinated cells to any $K \geq 2$. The stationary distribution of the Markov process $X(t)$ is:

$$
\begin{gathered}
\pi(x)=\pi(0)\left(\begin{array}{c}
x_{0}+x_{1} \ldots+x_{K} \\
x_{0}
\end{array}\right) \\
\times \prod_{k=0}^{K}\left(\begin{array}{c}
x_{k} \\
x_{1 k}, \ldots, x_{N_{k} k}
\end{array}\right) \rho_{1 k}^{x_{1 k}} \ldots \rho_{N_{k} k}^{x_{N_{k} k}},
\end{gathered}
$$

under the stability condition $\rho<1$, with

$$
\pi(0)=\frac{\left(1-\rho_{0}-\rho_{1}\right)\left(1-\rho_{0}-\rho_{2}\right) \ldots\left(1-\rho_{0}-\rho_{K}\right)}{\left(1-\rho_{0}\right)^{K-1}} .
$$

By (5)-(6), we deduce the mean throughput in the noncoordination zone $k$,

$$
\gamma_{k}=\mu_{k} \sigma\left(1-\rho_{0}-\rho_{k}\right),
$$

and the mean throughput in the coordination zone,

$$
\gamma_{0}=\mu_{0} \sigma\left(\sum_{k=1}^{K} \frac{1}{1-\rho_{0}-\rho_{k}}-\frac{K-1}{1-\rho_{0}}\right)^{-1} .
$$

\section{F. Priority to non-CoMP users}

Under this policy, non-CoMP users in each cell are scheduled first and are allocated all the radio resources whenever active; CoMP users wait untill resources in all coordinated cells become available. The stability condition is:

$$
\rho_{0}<\prod_{k=1}^{K}\left(1-\rho_{k}\right)
$$

which is more restrictive than the natural stability condition $\rho<1$. This is due to the fact that some cells may be idle when serving non-CoMP users.

The mean throughput in non-coordination zone $k$ is

$$
\gamma_{k}=\mu_{k} \sigma\left(1-\rho_{k}\right)
$$

There is no explicit expression for the mean throughput in the coordination zone.

\section{G. Priority to CoMP users}

Under this policy, CoMP users are scheduled first and are allocated all radio resources whenever active. Non-CoMP users are served only when there are no active CoMP users. The stability condition is the natural condition $\rho<1$. Since CoMP users are not affected by non-CoMP users, we have

$$
E\left(X_{0}\right)=\frac{\rho_{0}}{1-\rho_{0}} .
$$

By (5), the mean throughput in the coordination zone is:

$$
\gamma_{0}=\mu_{0} \sigma\left(1-\rho_{0}\right) \text {. }
$$

Applying known results of queuing theory [19], the mean number of flows in non-coordination zone $k$ is

$$
E\left(X_{k}\right)=\frac{\rho_{k}}{1-\rho_{0}}\left(1+\frac{\rho_{k}+\rho_{0} \mu_{k} / \mu_{0}}{1-\rho_{k}-\rho_{0}}\right)
$$

We deduce the mean throughput in non-coordination zone $k$,

$$
\gamma_{k}=\mu_{k} \sigma \frac{\left(1-\rho_{k}-\rho_{0}\right)\left(1-\rho_{0}\right)}{\left(1-\rho_{k}-\rho_{0}\right)+\rho_{k}+\rho_{0} \mu_{k} / \mu_{0}} .
$$

Observe that the mean throughput of CoMP users is positive whenever $\rho_{0}<1$ while the mean throughput of nonCoMP users in zone $\mathrm{k}$ is positive whenever $\rho_{0}+\rho_{k}<1$.

\section{H. Numerical results}

Figure 3 compares the throughput performance of the four policies for $K=2$ coordinated cells. We assume that there is only one region in each zone with $p_{0}=p_{1}=p_{2}=1 / 3$, $\mu_{1}=2, \mu_{2}=3 / 2$ and $\sigma=1$. This is the case of $\delta P=12 d B$ : the threshold for the difference in the received power between the cooperating cells, and a $50 \%$ coordination gain. We take $\phi_{0}=1 / 2$ for the static allocation (SA). Observe that proportional fairness (PF) outperforms the three other strategies. The priority to CoMP (Pri-C) strategy largely outperforms the priority to non-CoMP (Pri-NC) strategy at high load. In terms of stability, the Pri-NC strategy is the worst. This is due to the fact that prioritizing Non-CoMP users adversely affects the stability condition of CoMP users. The SA policy benefits from low complexity at the expense of degraded performance. The maximum load is approximately equal to 0.77 under the PriNC scheme and to 0.88 under the SA scheme, in accordance with the presented results. 


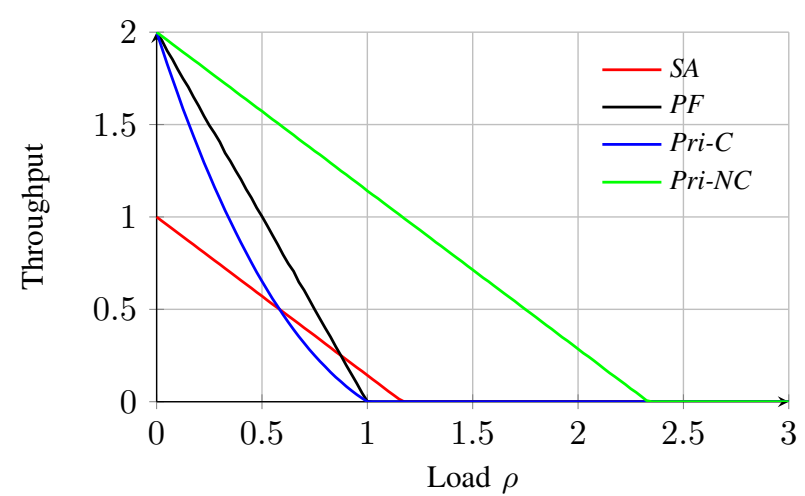

(a) Mean throughput of non-CoMP users

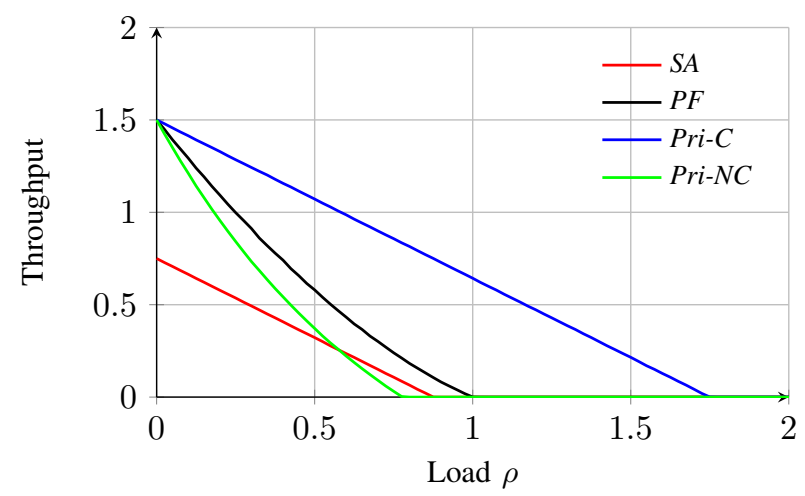

(b) Mean throughput of CoMP users

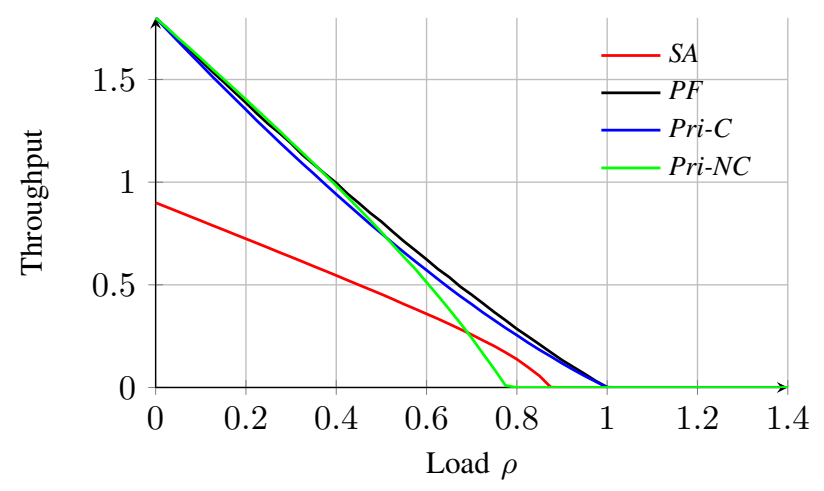

(c) Mean throughput in the cluster

Fig. 3. Throughput performance for two coordinated cells without mobility.

\section{IMPACT OF MOBILITY}

In this section, we add mobility to the previous model. We use the same mobility model as in [10]. Users move from a non-coordination zone of a cell to a non-coordination zone of another cell through the coordination zone, as shown in Figure 4 for two coordinated cells.

\section{A. Mobility model}

1) Inter-zone mobility: We assume that each user in region $N_{k}$ of non-coordination zone $k$ moves to region $N_{0}$ of the coordination zone and vice versa after exponential durations, at respective rates $\nu_{k, 0}$ and $\nu_{0, k}$. In state $x$, the total mobility rate from non-coordination zone $k$ to the coordination zone 0 and from coordination zone 0 to non-coordination zone $k$ are

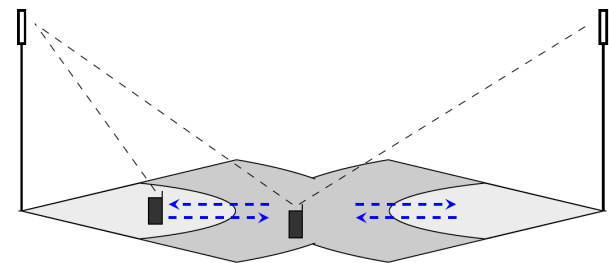

Fig. 4. A simple model with two coordinated cells and mobility.

equal to $x_{N_{k} k} \nu_{k, 0}$ and $x_{N_{0} 0} \nu_{0, k}$, respectively.

2) Intra-zone mobility: We assume that in each zone $k$, each user in region $i$ moves to region $i-1$ (for $i>1$ ) and to region $i+1$ (for $i<N_{k}$ ) after exponential durations, at respective rates $\nu_{i, i+1}^{(k)}$ and $\nu_{i, i-1}^{(k)}$. In state $x$, the total mobility rate from region $i$ to region $i+1$ and from region $i+1$ to region $i$ (for $i<N_{k}$ ) in zone $k$ are $x_{i k} \nu_{i, i+1}^{(k)}$ and $x_{i+1} \nu_{i+1, i}^{(k)}$, respectively. The probability that a user is in region $i$ of zone $k$ (for $k>0$ ) then satisfies

$$
q_{i k} \propto \frac{\nu_{0, k}}{\nu_{k, 0}} \prod_{j=i}^{N_{k}-1} \frac{\nu_{j+1, j}^{(k)}}{\nu_{j, j+1}^{(k)}},
$$

while the probability that a user is in region $i$ of zone $k=0$ satisfies

$$
q_{i 0} \propto \prod_{j=i}^{N_{0}-1} \frac{\nu_{j+1, j}^{(0)}}{\nu_{j, j+1}^{(0)}} .
$$

Note that

$$
\sum_{k=0}^{K} \sum_{i=1}^{N_{k}} q_{i k}=1
$$

The probability that a user is in zone $k$ is then given by:

$$
q_{k}=\sum_{i=1}^{N_{k}} q_{i k} .
$$

It is worth noting that, for mobile users, the traffic conservation equation (3) no longer applies: the traffic arriving in region $i$ of zone $k$ is not equal in general to the traffic served in region $i$ of zone $k$. Thus the mean throughput in region $i$ of zone $k$ is given by (2) but not by (4). Similarly, the mean throughput in zone $k$ is given by

$$
\gamma_{k}=\frac{E\left(\sum_{i} \mu_{i k} \sigma \phi_{k}(X) \frac{X_{i k}}{X_{k}}\right)}{E\left(X_{k}\right)},
$$

but not by (5). Now the overall traffic conservation equation in the cluster still applies so that the mean throughput in the cluster is still given by (6). It is still the arithmetic mean of the mean throughputs $\gamma_{k}$ weighted by the probability $p_{k}^{\prime}$ that an active user is in zone $k$, given by

$$
p_{k}^{\prime}=\frac{E\left(X_{k}\right)}{\sum_{j=0}^{K} E\left(X_{j}\right)}
$$

for all $k=0,1, \ldots, K$. 


\section{B. Stability condition}

The stability condition follows from the limiting regime of infinite mobility where $\nu_{i, i+1}^{(k)}, \nu_{i+1, i}^{(k)} \rightarrow \infty$ for all $i<N_{k}$ and $k \geq 0$, and $\nu_{k, 0}, \nu_{0, k} \rightarrow \infty$ for all $k>0$ [12]. In this regime, the mean service rate in zone $k$ becomes

$$
\bar{\mu}_{k}=\sum_{i} q_{i k} \mu_{i k} / q_{k} .
$$

The overall mean service rate is

$$
\bar{\mu}=\left(1-\phi_{0}\right) \sum_{k=1}^{K} \bar{\mu}_{k}+\phi_{0} \bar{\mu}_{0}
$$

under the SA scheme,

$$
\bar{\mu}=\left(1-q_{0}\right) \sum_{k=1}^{K} \bar{\mu}_{k}+q_{0} \bar{\mu}_{0}
$$

under the PF scheme,

$$
\bar{\mu}=\sum_{k=1}^{K} \bar{\mu}_{k}
$$

under the Pri-NC scheme, and

$$
\bar{\mu}=\bar{\mu}_{0}
$$

under the Pri-C scheme. The stability condition under the four schemes is

$$
\rho<\bar{\mu} / \mu .
$$

Note that in the absence of inter-zone mobility, the stability condition is:

$$
\rho<\left(\frac{p_{0}}{\mu_{0}}+\max _{k=1, \ldots, K} \frac{p_{k}}{\mu_{k}}\right) \min _{k=0, \ldots, K} \frac{\phi_{k} \bar{\mu}_{k}}{p_{k}}
$$

under the SA scheme,

$$
\rho<\left(\frac{p_{0}}{\mu_{0}}+\max _{k=1, \ldots, K} \frac{p_{k}}{\mu_{k}}\right)\left(\frac{p_{0}}{\bar{\mu}_{0}}+\max _{k=1, \ldots, K} \frac{p_{k}}{\bar{\mu}_{k}}\right)^{-1}
$$

under the PF and Pri-C schemes, and

$$
\frac{\mu_{0}}{\bar{\mu}_{0}} \rho_{0}<\prod_{k=1}^{K}\left(1-\frac{\mu_{k}}{\bar{\mu}_{k}} \rho_{k}\right),
$$

under the Pri-NC scheme, with

$$
\bar{\mu}_{k}=\sum_{i} q_{i k} \mu_{i k}
$$

and

$$
q_{i k} \propto \prod_{j=i}^{N_{k}-1} \frac{\nu_{j+1, j}^{(k)}}{\nu_{j, j+1}^{(k)}}
$$

\section{Throughput in light traffic}

The performance in light traffic (that is, when $\rho \rightarrow 0$ ) is the same for all policies except the static allocation: a user when alone in the system is always allocated all radio resources. For instance, we consider that there is only one region in each zone. The probability that a user in non-coordination zone $k$ moves to the coordination zone before leaving the system is

$$
\alpha_{k, 0}=\frac{\nu_{k, 0}}{\mu_{k}+\nu_{k, 0}} .
$$

Similarly, the probability that a user in the coordination zone moves to non-coordination zone $k$ before leaving the system is

$$
\alpha_{0, k}=\frac{\nu_{0, k}}{\mu_{0}+\nu_{0}},
$$

where

$$
\nu_{0}=\sum_{j=1}^{K} \nu_{0, j} .
$$

The mean duration of a flow initiated in a non-coordination zone is

$$
\begin{aligned}
& T_{k}=\frac{1}{\nu_{k, 0}+\mu_{k}} \\
&+\frac{\alpha_{k, 0}}{1-\sum_{j=1}^{K} \alpha_{0, j} \alpha_{j, 0}}\left[\frac{1}{\nu_{0}+\mu_{0}}+\sum_{j=1}^{K} \frac{\alpha_{0, j}}{\nu_{j, 0}+\mu_{j}}\right],
\end{aligned}
$$

while the mean duration of a flow initiated in the coordination zone is given by:

$$
T_{0}=\frac{1}{1-\sum_{j=1}^{K} \alpha_{0, j} \alpha_{j, 0}}\left[\frac{1}{\nu_{0}+\mu_{0}}+\sum_{j=1}^{K} \frac{\alpha_{0, j}}{\nu_{j, 0}+\mu_{j}}\right] .
$$

We deduce the mean duration of a flow initiated in the cluster,

$$
T=\sum_{k=0}^{K} p_{k} T_{k},
$$

and the average throughput in light traffic $\gamma=\sigma / T$.

Under the SA policy, we get the mean throughput similarly by replacing $\mu_{k}$ by $\phi_{k} \mu_{k}(\forall k \leq K)$.

\section{Numerical results}

Consider the same scenario of two coordinated cells as in the previous section, with $\nu_{01}=\nu_{10}=\nu_{02}=\nu_{20}=1$. The results are obtained by the numerical evaluation of the stationary distribution of the Markov process $X(t)$ and shown in Figure 5.

We observe that inter-zone mobility improves the mean throughput in the cluster for all strategies except for the Pri-C strategy. Under this strategy, mobility leads to a throughput degradation both for non-CoMP users and for CoMP users. This is due to the fact that non-CoMP users may then suffer from bad radio conditions (served with CoMP mode) leading to extra waste of resources. Based on this observation, the following conclusion may be drawn: it is not worth losing a resource to serve a CoMP user if that user is moving to the center and could benefit from better channel conditions. The strategy of prioritizing non-CoMP users proves to be the best strategy when users move. This can be explained by the fact 
that prioritizing Non-CoMP users gives the chance to celledge users to move and to be served in better radio conditions where coordination is not required. However, according to the numerical and analytical results the PF strategy remains a good compromise in a network where the users' mobility cannot be predicted. In accordance with the presented results, the maximum loads are approximately equal to $1.07,1.23$, 1.55 and 0.6 under the SA, PF, Pri-NC and Pri-C strategies, respectively. This shows that in terms of stability Pri-NC is the best strategy while Pri-C is the worst.

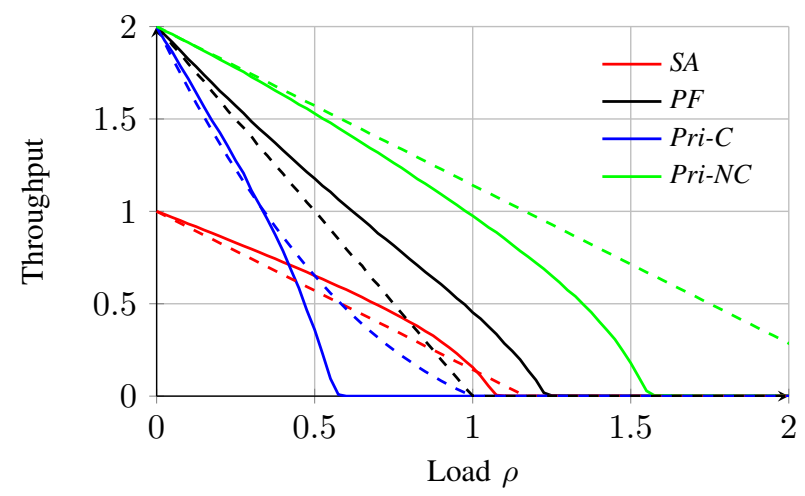

(a) Mean throughput of non-CoMP users

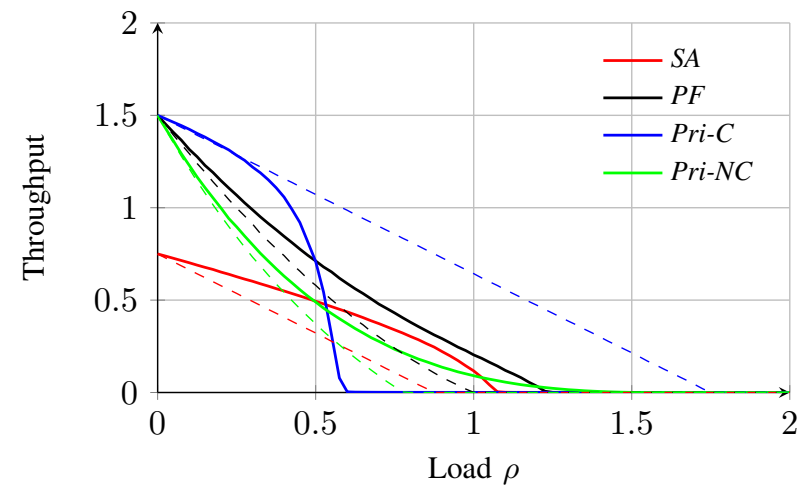

(b) Mean throughput of CoMP users

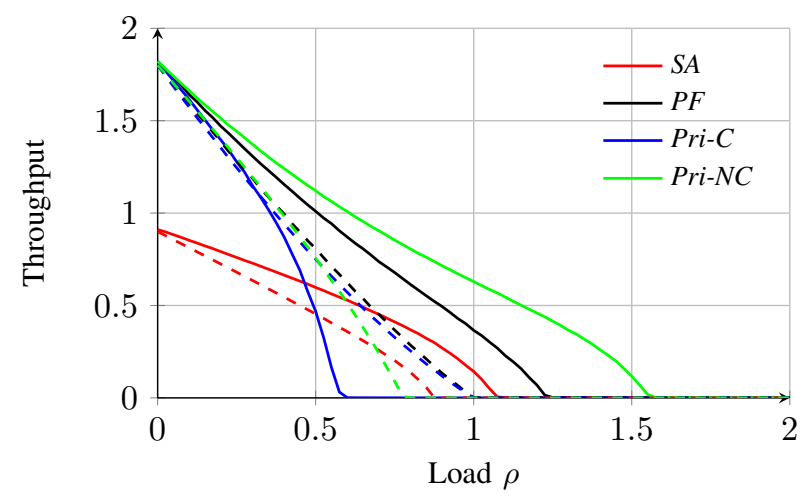

(c) Mean throughput in the cluster

Fig. 5. Throughput performance for two coordinated cells with mobility (solid line) and without mobility (dashed line).

\section{CONCLUSION}

This paper studies the impact of mobility on the performance of cell coordination, showing the critical impact of the scheduling strategy. By prioritizing CoMP users, mobility surprisingly incurs a throughput degradation. The proportional fairness strategy is a good compromise when the mobility of users is not known a priori. However, when mobility can be predicted accurately, it is better to prioritize non-CoMP users. Following this conclusion, we plan to consider more advanced scheduling strategies in the future. We shall also consider other CoMP techniques such as coordinated beamforming [20].

\section{REFERENCES}

[1] 3GPP TR 36.913, "Requirements for Further Advancements for EUTRA (LTE-Advanced),"

[2] R. Irmer, H. Droste, P. Marsch, M. Grieger, G. Fettweis, S. Brueck, H. Mayer, L. Thiele, and V. Jungnickel, "Coordinated multipoint: Concepts, performance, and field trial results," IEEE Communications Magazine, vol. 49, no. 2, pp. 102-111, 2011.

[3] 3GPP R1-083569, "Further Discussion on Inter-Cell Interference Mitigation Through Limited Coordination ," 2008.

[4] A. Khlass, T. Bonald, and S. Elayoubi, "Flow-level performance of intra-site coordination in cellular networks," in IEEE WiOpt, Tsukuba Japan, May 2013, pp. 216-223, 2013.

[5] 3GPP R1-090613, "Discussion on CoMP-SU-MIMO," 2009.

[6] 3GPP R1-091415, "Further discussion of frequency plan scheme on CoMP-SU-MIMO," 2009.

[7] J. Liu, Y. Chang, Q. Pan, X. Zhang, and D. Yang, "A novel transmission scheme and scheduling algorithm for CoMP-SU-MIMO in LTE-A system," in Proceedings of the 71st IEEE VTC, 16-19 May 2010, Taipei, Taiwan, pp. 1-5, 2010.

[8] S. Brueck, L. Zhao, J. Giese, and M. A. Amin, "Centralized Scheduling for Joint Transmission Coordinated Multi-Point in LTE-Advanced," in Proc. ITG/IEEE Workshop on Smart Antennas, , 23-24 Feb 2010, Bremen, Germany, 2010.

[9] W. Zhou, Y. Zhang, P. Qin, W. Chen, and X. Li, "Joint scheduling algorithms for LTE-A comp system," JCP, vol. 8, no. 11, pp. 27952801, 2013.

[10] N. Abbas, T. Bonald, and B. Sayrac, "Opportunistic gains of mobility in cellular data networks," in IEEE WiOpt, Mumbai India, 2015.

[11] T. Bonald, S. C. Borst, and A. Proutière, "How mobility impacts the flow-level performance of wireless data systems," in Proceedings IEEE INFOCOM 2004, Hong Kong, China, March 7-11, 2004.

[12] T. Bonald, S. C. Borst, N. Hegde, M. Jonckheere, and A. Proutiere, "Flow-level performance and capacity of wireless networks with user mobility," Queueing Syst., vol. 63, no. 1-4, pp. 131-164, 2009.

[13] S. C. Borst, A. Proutière, and N. Hegde, "Capacity of wireless data networks with intra- and inter-cell mobility," in INFOCOM. 23-29 April 2006, Barcelona, Catalunya, Spain, 2006.

[14] S. C. Borst, N. Hegde, and A. Proutiere, "Mobility-driven scheduling in wireless networks," in INFOCOM. 19-25 April 2009, Rio de Janeiro, Brazil, pp. 1260-1268, 2009.

[15] M. K. Karray, "User's mobility effect on the performance of wireless cellular networks serving elastic traffic," Wireless Networks, vol. 17, no. 1 , pp. 247-262, 2011.

[16] J. Kim, B. Kim, J. Kim, and Y. H. Bae, "Stability of flow-level scheduling with markovian time-varying channels," Perform. Eval., vol. 70, no. 2, pp. 148-159, 2013.

[17] T. Bonald and M. Feuillet, Network Performance Analysis. ISTE/Wiley, 2011.

[18] F. P. Kelly, A. K. Maulloo, and D. Tan, "Rate control for communication networks: shadow prices, proportional fairness and stability," Journal of the Operational Research Society, vol. 49, pp. 237-252, 1998.

[19] L. Kleinrock, Queueing Systems, vol. I: Theory. Wiley Interscience, 1975.

[20] 3GPP TR 36.819, “ Coordinated Multi-point Operation for LTE Physical Layer Aspects," 2011-2012. 